ÉGYPTE

monde arabe

\section{Égypte/Monde arabe}

6 | 2003

D'une intifâda l'autre. La Palestine au quotidien

\title{
Le processus de paix au Moyen-Orient et l'autorité de la loi
}

Deux objectifs inconciliables?

Lynn Welchman

\section{OpenEdition}

\section{Journals}

Édition électronique

URL : https://journals.openedition.org/ema/957

DOI : $10.4000 /$ ema.957

ISSN : 2090-7273

Éditeur

CEDEJ - Centre d'études et de documentation économiques juridiques et sociales

Édition imprimée

Date de publication : 31 décembre 2003

Pagination : 207-231

ISBN : 2-87027-993-0

ISSN : $1110-5097$

\section{Référence électronique}

Lynn Welchman, «Le processus de paix au Moyen-Orient et l'autorité de la loi », Égypte/Monde arabe

[En ligne], 6| 2003, mis en ligne le 08 juillet 2008, consulté le 07 juillet 2022. URL : http://

journals.openedition.org/ema/957 ; DOI : https://doi.org/10.4000/ema.957

Ce document a été généré automatiquement le 7 juillet 2022.

Tous droits réservés 


\title{
Le processus de paix au Moyen- Orient et l'autorité de la loi
}

Deux objectifs inconciliables?

\author{
Lynn Welchman
}

1 Cet article se propose de donner un aperçu des différents aspects du droit international humanitaire liés à la politique étrangère de l'Union européenne (UE) concernant le processus de paix au Moyen-Orient, avec des exemples précis sur le comportement du Royaume-Uni. Ce faisant, il cherche à étudier la disparité entre les promesses des États tiers de faire respecter le droit international humanitaire et les impératifs des agendas politiques adoptés par ces États. Dans le contexte du processus de paix au MoyenOrient, l'impératif politique déclaré de l'UE est d'aider à résoudre le conflit en apportant son appui à un processus dans lequel les États-Unis sont le principal tiers acteur étatique. En même temps, les États membres de l'UE affirment leur engagement à garantir le respect du droit, compte tenu surtout de leurs " positions de principe " insistant sur le fait qu'Israël doit se conformer au droit international humanitaire dans tous les territoires qu'il occupe. Cependant, il est clair que l'action entreprise pour soutenir le processus de paix et faire respecter le droit international a été subordonnée aux exigences du processus politique de la « période intérimaire » qui devait conduire aux négociations sur le statut définitif de la Palestine.

2 Une attention particulière sera portée à la manière dont l'UE conçoit le principe de la responsabilité des États tiers dans les situations d'occupation militaire. Bien que cela s'applique généralement aussi aux questions du Golan et du Sud-Liban, l'accent sera mis sur les réponses apportées à des situations particulières engendrées, premièrement par l'intifâda qui a débuté en 1987 dans les Territoires palestiniens occupés, deuxièmement, durant le processus de paix, dans la période antérieure à l'Accord intérimaire conclu entre Israël et l'Organisation de libération de la Palestine (OLP en 1993) 'et, troisièmement, au cours de la période 1997-1999. Enfin, une quatrième partie s'intéressera aux évolutions de la question depuis le déclenchement de la seconde intifâda. Le présent article fait référence aux déclarations de l'UE et aux résolutions du Conseil de sécurité de l'Onu relatives aux responsabilités qui incombent aux États tiers 


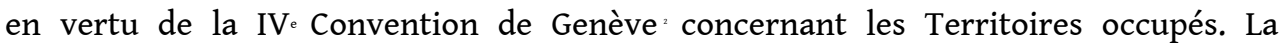
première résolution évoquée concerne l'obligation pour toutes les hautes parties contractantes d' « assurer le respect » de ces dispositions en toutes circonstances', ce qui signifie, selon Pictet, dans son commentaire de la IV $\mathrm{V}^{\mathrm{C}}$ Convention de Genève, que les États parties «ne doivent pas se contenter d'appliquer ces dispositions eux-mêmes, mais devraient faire tout ce qui est en leur pouvoir pour s'assurer que les principes humanitaires sous-jacents à la Convention sont appliqués à l'échelle universelle " (Pictet, 1958, p. 15 ; cAzâm, 1997 ; Stephens, 1989). La seconde, fondée sur ce principe, est l'obligation, pour toutes les hautes parties contractantes, de prendre toute mesure législative nécessaire pour fixer les sanctions pénales adéquates à appliquer aux personnes ayant commis ou donné l'ordre de commettre l'une ou l'autre des infractions graves à la présente Convention - c'est-à-dire des crimes de guerre --, de les rechercher et de les déférer à leurs propres tribunaux, quelle que soit leur nationalité. En 1977, l'article $89 \mathrm{du}$ premier Protocole additionnel à la Convention de Genève développe le principe de la responsabilité de la tierce partie dans la mise en œuvre de ces dispositions en obligeant les hautes parties contractantes à agir, tant conjointement que séparément, dans les cas de violations graves de la Convention ou du présent Protocole, en coopération avec l'Organisation des Nations unies et conformément à la Charte des Nations unies.

3 Il faut d'abord remarquer que les États membres de l'UE, ainsi que d'autres États de la communauté internationale, ont reconnu le rôle des États tiers dans le maintien du respect du droit durant les périodes d'occupation militaire - et ce, en s'assurant de la mise en œuvre de la IV ${ }^{\cdot}$ Convention de Genève et du droit coutumier international par la puissance occupante. Il faut ensuite constater que, malgré la cohérence des déclarations et de la politique des États membres de l'UE - et souvent leur insistance sur la nécessité que la Convention soit appliquée dans les Territoires palestiniens occupés durant l'occupation israélienne -, ces pays n'ont pas réussi à mener une action efficace dans le cadre de leurs relations bilatérales avec Israël permettant de faire respecter les dispositions du droit international et, par conséquent, la protection des populations civiles.

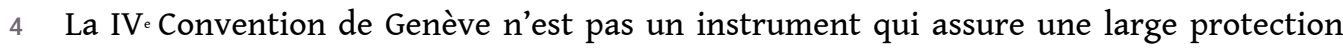
des droits de l'homme. En fait, elle ne fait que garantir une protection minimale aux populations civiles sous occupation en condamnant les violations des droits de l'homme pour raisons politiques qu'une puissance occupante serait tentée de commettre. La Convention se fonde sur le droit coutumier international en vertu duquel tout programme d'annexion territoriale est illégal. Correctement appliquée et mise en œuvre, la Convention préserve ainsi les perspectives de paix et de réconciliation en assurant un degré minimum de protection des droits de l'homme et en évitant les impasses auxquelles conduisent les politiques fondées sur la conquête et l'annexion. Dans son résumé des objectifs des « lois relatives à l'occupation », Roberts dit qu'elles tendent à " ouvrir des perspectives pour un éventuel accord de paix» et ajoute que : "L'interdiction d'annexer les territoires occupés et les règles s'opposant au transfert de populations de et vers les Territoires occupés reflètent en partie cet objectif.» (Roberts, 1992, p. 28).

5 Le fait de ne pas respecter ou faire respecter le droit a des répercussions aussi bien sur la protection des droits de l'homme que sur les espoirs de résoudre le conflit. La relation cruciale entre le respect du droit international humanitaire et les perspectives 
de promotion et de soutien d'un processus de paix visant une solution juste et durable au conflit a été explicitement reconnue par les États membres de l'UE. En 1990, le représentant de la Grande-Bretagne à la Commission des Nations unies aux droits de l'homme s'est exprimé en ces termes :

«Le respect des droits de l'homme est également utile sur le plan pratique. Sans lui, il ne peut exister de paix durable, ni au Moyen-Orient ni nulle part ailleurs. Ceci s'applique aux Territoires occupés plus qu'à n'importe quelle autre région au monde. [...] Avant qu'une paix durable ne puisse se réaliser, il est indispensable d'instaurer un climat de plus grande confiance mutuelle entre les Israéliens et les Palestiniens dans les Territoires occupés que ce qui - malheureusement - règne à présent. Là aussi, la solution réside dans le respect des droits de l'homme et la complète conformité d'Israël à ses obligations internationales en la matière. L'obstacle réel à l'instauration d'un tel climat est qu'Israël persiste dans son refus de reconnaitre que les dispositions des accords internationaux relatifs à la question s'appliquent aux territoires qu'elle occupe.».

6 En dépit de l'unanimité de la communauté internationale (Roberts, 1992, p. 52), les gouvernements successifs d'Israël refusent de reconnaitre l'applicabilité de jure de la Convention à tous les Territoires occupés par Israël depuis 1967 (Shehadeh, 1985, p. 11.). Les mécanismes de mise en œuvre internes à la convention, par la désignation d'une puissance protectrice, sont donc demeurés inopérants'. De la même manière, le fait que, malgré ce consensus, le mécanisme de protection du droit international humanitaire ne soit toujours pas appliqué dans les Territoires palestiniens occupés est la conséquence directe du choix des principaux États tiers de ne pas faire pression pour satisfaire aux exigences de ce droit, et ce, dans le but d'influencer et d'orienter le processus politique dans lequel ils se sont investis et d'imposer leur propre politique comme une condition sine qua non. Cet échec pourrait être perçu comme ayant considérablement compliqué les perspectives de règlement du conflit. La tolérance de facto sur la non-application du droit international humanitaire a conduit à la « création de faits » en violation de la Convention - notamment les colonies et l'annexion de Jérusalem-Est - qui appartiennent aux questions les plus difficiles et apparemment insolubles de l'ordre du jour des négociations entre les parties. Comme le fait remarquer Campbell : « Lorsque les implications du droit international humanitaire sur le statut définitif sont examinées, deux thèmes surgissent immédiatement : les colonies et Jérusalem-Est.» (Campbell, 1996, p. 52). La discussion de ces deux principales violations (toutes deux au cœur des prohibitions d'annexion) est reportée, avec d'autres questions, à la phase des négociations sur le statut définitif. Quel que soit le statut légal des accords entre Israël et l'OLP et leur effet sur la situation des territoires durant la période intérimaire (Campbell, 1996, p. 43-47), ces accords ne peuvent sûrement pas remplacer le droit international humanitaire auquel sont soumis, entre autres, les États membres de l'UE et leur position concernant Jérusalem, les colonies et autres questions relatives à l'occupation. La Déclaration de principes et les accords ultérieurs, qui mentionnent spécifiquement l'application des résolutions 242 et $338 \mathrm{du}$ Conseil de sécurité comme base du règlement permanent, font figurer dans l'accordcadre l'interdiction d'acquérir des territoires par la guerre". Néanmoins, comme le dit Campbell à propos de la période de transition :

«Alors que les progrès politiques bénéficiant à une partie du peuple palestinien sont indéniables, les normes juridiques qui ébranlent les affirmations israéliennes ont été ignorées. Les nombreuses questions non résolues trouveront peut-être une solution satisfaisante lors des négociations sur le statut définitif. Cependant, les chances pour que cela se produise seraient nettement plus grandes si le processus 
de transition était plus clairement fondé sur les normes juridiques de la IV

Convention de Genève » (Campbell, 1993, p. 53)".

7 Avant le début du processus de paix en cours, l'UE jouait un rôle politique primordial pour « assurer le respect» de la Convention dans les Territoires palestiniens occupés. Durant les premières années de l'intifâda, déclenchée en décembre 1987 en Cisjordanie et dans la bande de Gaza, plusieurs facteurs ont contribué à pousser certains États européens à envisager une action fondée sur le respect du droit international dans les Territoires occupés. Ce développement s'est traduit par l'adoption de la Déclaration de Dublin par le Conseil européen ainsi que de la résolution 681 du Conseil de sécurité (1990). Cependant, en 1991, à la fin de la guerre du Golfe, les efforts des États-Unis en vue d'une conférence de paix dans la région ont été perçus comme une demande de suspension de toute autre discussion sur les mesures à prendre pour faire respecter le droit international en tant que mécanisme approprié pour assurer les droits de l'homme dans les Territoires palestiniens et y promouvoir la solution du conflit.

Vers une action fondée sur la loi : La résolution 681 du Conseil de sécurité (1990)

8 En décembre 1987, deux semaines après le début de l'intifâda, le Conseil de sécurité a adopté la résolution 605 qui engage Israël à se conformer «immédiatement et scrupuleusement " à la IVe Convention de Genève « et à renoncer sur-le-champ à ses politiques et pratiques qui constituent une violation des dispositions de la Convention ». Conscient de «la nécessité d'envisager des mesures visant à assurer la protection impartiale de la population civile palestinienne, le Conseil de sécurité a demandé au Secrétaire général de lui présenter un rapport sur la question». En s'engageant vis-à-vis des évènements dans les Territoires occupés, le Conseil de sécurité de l'Onu et les États membres de l'UE s'étaient donc préoccupés, depuis le début de l'intifâda, de la question d'une protection internationale fondée sur le droit.

9 Le rapport du Secrétaire général, publié dans le contexte de la déportation des Palestiniens hors des Territoires et d'une longue liste de préjudices, a mis l'accent sur les moyens et les mesures permettant d'assurer la sécurité et la protection de la population civile palestinienne. Y fut incluse une liste des violations israéliennes de la Convention ayant déjà spécifiquement «fait l'objet de nombreuses résolutions du Conseil de sécurité ». Attirant l'attention sur la position unanime du Conseil de sécurité et de l'Assemblée générale de l'Onu concernant l'applicabilité de la Convention, la principale recommandation du Secrétaire général au Conseil de sécurité était la suivante :

«Le moyen le plus efficace d'assurer la sécurité et la protection de la population civile dans les Territoires occupés serait donc, pour Israël, d'appliquer pleinement les dispositions de la IV. Convention. À cette fin, je recommande que le Conseil de sécurité envisage de lancer un appel formel aux hautes parties contractantes à la IV. Convention de Genève qui entretiennent des relations diplomatiques avec Israël, focalisant leur attention sur l'obligation qu'ils ont, en vertu de l'Article I de la Convention, "de respecter et faire respecter la présente Convention en toutes circonstances" et de les presser de mettre en œuvre tous les moyens en leur disposition pour persuader le gouvernement israélien de changer sa position concernant l'applicabilité de la convention. »

10 Bien que le Conseil ait fait référence, dans les déclarations de son président", à l'obligation pour les États parties, de "faire respecter la Convention», il a fallu attendre la fin de 1990 pour qu'un tel appel aux hautes parties contractantes soit inclus dans une résolution". 
11 Durant cette période intermédiaire, les États-Unis ont opposé leur veto à un projet de résolution au Conseil de sécurité connu plus tard comme la " résolution de protection » (Protection Resolution). En mai 1990, dans le contexte des divers dommages subis par les Palestiniens, le Conseil de sécurité a été convoqué pour étudier le projet de création d'une commission constituée de trois de ses membres chargée « d'examiner la situation au vu des politiques et des pratiques d'Israël, puissance occupante », et de soumettre au Conseil ses recommandations concernant « les moyens et les mesures à prendre en vue d'assurer la sûreté et la protection des civils palestiniens sous l'occupation israélienne ${ }^{*}$. Le texte du projet renvoie explicitement à la réaffirmation préliminaire de l'applicabilité de la IV Convention de Genève. À cette résolution, les États-Unis ont opposé leur veto et leur ambassadeur a déclaré que : «Les États-Unis auraient soutenu des mesures répondant à la montée en spirale des troubles. Mais nous avons clairement dit que ces mesures ne devaient pas retarder les efforts destinés à reprendre le processus de paix dans les plus brefs délais. "'Par contre, les États de l'UE membres au Conseil de sécurité ont saisi l'occasion de la fin de la déclaration du président du Conseil, à la fin juin 1990, pour rappeler particulièrement l'obligation pour les États tiers de faire respecter la IV Convention de Genève. Se déclarant préoccupés du fait que «les droits de l'homme continuent à être insuffisamment respectés dans les Territoires occupés ", le Conseil européen appelle à une nouvelle action, conformément à la Convention, pour assurer cette protection. " "

12 Peu de temps après, en août 1990, avec l'invasion du Kuwît par l'Irâq, l'attention sur les évènements au Moyen-Orient s'est centrée sur une toute autre question et la communauté internationale s'est engagée dans la préparation de la «Tempête du désert ». Une série de résolutions du Conseil de sécurité s'est succédée rapidement, faisant référence à la Convention de Genève ainsi qu'au chapitre viI de la Charte des Nations unies. Au début du mois d'octobre, le Conseil de sécurité a été convoqué pour étudier la situation dans les Territoires palestiniens occupés à la suite de l'assassinat de dix-sept civils palestiniens par les forces de sécurité israéliennes au Dôme du Rocher. Deux résolutions ont suivi et il a été demandé au Secrétaire général de présenter un rapport. L'examen de ce rapport a eu lieu sur fond des résolutions relatives à l'occupation du Kuwît par l'Irâq. Les membres du Conseil étaient en effet confrontés au refus de l'Irâq de reconnaître l'applicabilité de la IVe Convention de Genève, son occupation du Kuwît et des preuves de graves violations commises contre la population civile de ce pays. Dans ses résolutions, le Conseil de sécurité rappelait à l'Irâq ses obligations en vertu de la Convention en ce qui concerne les infractions graves commises par ses agents et invitait tous les États à "rassembler les informations fondées qui se trouvent en leur possession ou leur sont fournies concernant les infractions graves commises par l'Irâq... et à les communiquer au Conseil de sécurité. »".

Les comparaisons qui ont été faites entre la réaction des puissances occidentales membres du Conseil de sécurité à l'occupation du Kuwît par l'Irâq et leur réaction à l'occupation de longue date des Territoires palestiniens - bien que n'ayant pas été admises comme comparaisons légitimes - ont sûrement augmenté la pression politique s'exerçant sur Israël pour qu'il rende compte de ses agissements, et ceci en particulier du fait de l'engagement des États arabes dans la coalition internationale contre l'Irâq. Enfin, la goutte d'eau qui a fait déborder le vase et permis l'adoption d'une résolution du Conseil de sécurité faisant mention d'une action des États tiers conformément au 
droit international, a été l'annonce, par les autorités israéliennes, des ordres de déportation de quatre Palestiniens de Gaza.

La déportation de civils des Territoires occupés par une puissance occupante est en effet considérée, aux termes de la Convention, comme une violation grave de ses dispositions". Cependant, ni le Conseil de sécurité ni l'UE n'avaient utilisé ce langage concernant la politique de déportation pratiquée par Israël, bien qu'ils aient souvent condamné fermement les déportations en tant que violation de la Convention ${ }^{*}$. Par conséquent, dans le climat politique particulier qui régnait en décembre 1990, la résolution 681 (1990) du Conseil de sécurité est une innovation et indique la prise en considération sérieuse d'une action à mener, conformément au droit, pour réprimer la violation par Israël du droit international humanitaire. Pour la première fois, la résolution inclut un appel direct aux hautes parties contractantes à ladite Convention, les incitant à exiger d'Israël le respect de la IV. Convention de Genève. La même résolution charge le Secrétaire général de convoquer une réunion des hautes parties contractantes à ladite Convention, d'examiner les «mesures éventuelles» qui pourraient être prises conformément à la Convention et lui confie, en outre, les responsabilités incombant au Conseil de sécurité concernant le suivi de la situation des civils palestiniens soumis à l'occupation israélienne.

Assumant ses responsabilités, le Secrétaire général a fait circuler, à la suite de l'arrêt des hostilités dans le Golfe en mars 1991, une note verbale destinée aux États membres. Son premier rapport présenté au Conseil de sécurité conformément à la résolution 681 (1990) date d'avril 1991". En réponse à sa demande de renseignement, le Luxembourg, alors président du Conseil européen, a déclaré que les États membres soutenaient le principe d'une réunion des hautes parties contractantes pour examiner les mesures qu'il conviendrait de prendre pour protéger les droits de la population civile, mais que de telles mesures ne devraient s'appliquer qu'au «moment propice». Ils se sont dits prêts à appuyer tout effort visant l'application de ladite Convention dans les Territoires occupés et qu'ils examineraient la constitution d'un « comité consultatif » qui pourrait étudier les moyens de sa mise en œuvre ${ }^{x}$. Il apparaît, par conséquent, que les États de l'UE envisageaient sérieusement d'entreprendre une action conformément au droit pour remédier aux abus d'Israël contrevenant au droit humanitaire dans les Territoires palestiniens.

En dehors du cadre du droit international : la présence internationale temporaire à Hébron (Tiph)

16 Toutefois, au moment où les réponses à la note verbale du Secrétaire général ont été envoyées, le contexte politique connaissait un changement radical du fait du contrecoup de la guerre du Golfe. Le Secrétaire d'État américain, James Baker, avait commencé sa navette diplomatique au Moyen-Orient et de fortes pressions étaient exercées de tous côtés pour entamer le processus de paix dans la région. Les tentatives de l'UE et d'autres pays européens pour une action visant à mettre le droit en vigueur, restaient en attente par respect pour l'initiative politique des États-Unis. L'idée d'une conférence réunissant les hautes parties contractantes devait ressusciter au cours de l'été 1997 lors de l'Assemblée générale des Nations unies débouchant, deux ans plus tard, sur une réunion de dix minutes immédiatement ajournée. En attendant, la convocation de la conférence de Madrid sur la paix, en 1991, marque le début d'une période où s'accentue le conflit constaté par les États acteurs entre la recherche 
rigoureuse de l'application du droit international et les délicats processus politiques de la diplomatie internationale dans la quête de solutions aux conflits.

Marginalisée dans les négociations, l'Organisation des Nations unies est demeurée inactive en tant que forum promouvant l'application du droit international. De son côté, la Communauté européenne de l'époque a été invitée à la Conférence de paix de Madrid en tant que participant, et non comme commanditaire, c'est-à-dire avec un statut semblable à celui des Nations unies. Ayant suspendu ses propres délibérations sur la politique à adopter pour la mise en œuvre du droit international humanitaire, le représentant de la Communauté européenne à la Conférence de Madrid a néanmoins fait une claire déclaration des principes sur lesquels se fondent certaines positions de la Communauté qui demeurent inchangées :

«Les principes directeurs des Douze dans le processus de négociation sont ceux qui ont longtemps guidé notre position. Ils sont demeurés inchangés. Ces principes sont ceux des résolutions 242 et 338 du Conseil de sécurité : le principe de la terre contre la paix, le droit de tous les États de la région, y compris Israël, de vivre dans des frontières sûres et reconnues et d'exercer leur droit à l'autodétermination. Notre position sur les questions relatives aux Territoires occupés, y compris JérusalemEst, est également bien connue. Un règlement global devrait, à notre avis, inclure ces principes. Mais nous ne prétendons pas prescrire la manière dont ils devraient être mis en œuvre sur le terrain... »"

La déclaration de la Communauté européenne recommande vivement la prudence des deux côtés et «engage Israël à respecter les dispositions de la IV convention de Genève ». Cependant, en dépit de cette politique de la Communauté européenne, Israël ne s'est pas engagé à s'acquitter de ses obligations en vertu du droit international et le processus de paix n'exigeait pas qu'il le fasse. La Communauté européenne a maintenu sa position, même pendant les crises telles que la déportation de près de quatre cents Palestiniens à travers la frontière libanaise en décembre 1992 (Welchman, 1996). En été 1993, Yitzhak Rabin et Yâsir 'Arafât annoncent officiellement leur reconnaissance mutuelle et, en septembre, la déclaration de principes est signée à Washington. L'autre question relative à la mise en application de l'autorité de la loi dans les Territoires palestiniens, notamment par l'extension de certaines formes de protection internationale de la population civile soumise à l'occupation, a été soulevée au début de 1994 à la suite du massacre commis par des colons israéliens contre des fidèles palestiniens en prière dans la mosquée d'Abraham à Hébron, massacre qui a causé la mort de plus de cinquante civils palestiniens et fait plusieurs centaines de blessés". La question des colonies a été amenée au cœur du débat de la manière la plus dramatique et les pourparlers de paix ont été suspendus. Au cours des discussions, il était clair que l'objectif premier des principaux États tiers était de revenir le plus vite possible au processus politique en vue d'accélérer la solution du conflit. Ceci impliquait cependant de ne pas aborder la question de la nature structurelle des violations du droit international qui avait directement suscité le problème à l'origine des discussions.

Dans les semaines qui suivirent le massacre, les États européens ont de plus en plus replacé les meurtres et les mesures envisagées en réponse à ces meurtres dans le contexte du processus de paix sans mentionner le droit et les obligations existantes. Ainsi, bien que différents États membres de l'UE aient confirmé que les colonies étaient une violation de la IVe Convention de Genève et réaffirmé les obligations d'Israël en vertu de ladite Convention, la déclaration du Conseil européen relative au processus de 
paix au Moyen-Orient fait référence à l'idée d'une protection et d'une "présence internationale » indépendamment de ces obligations :

«Alors que les récents évènements dramatiques à Hébron, déjà condamnés par l'UE, ne devraient pas interrompre le processus de paix au Moyen-Orient, le Conseil de l'UE:

- encourage la reprise des négociations du processus de paix entre toutes les parties et demande au Conseil de sécurité d'adopter rapidement une résolution appropriée ;

- accueille favorablement les mesures prises par le gouvernement d'Israël pour assurer la sécurité et la protection de tous les habitants des Territoires occupés ;

- demande aux parties de discuter la question de la sécurité des Palestiniens, y compris la question de certaines colonies, et d'accepter l'application des mesures appropriées ;

- soutient la mise en place d'une présence internationale dans les Territoires occupés laquelle l'UE se déclare disposée à participer".»

Pour sa part, le Conseil de sécurité a mis presque un mois pour faire adopter la résolution 904 (18 mars 1994) condamnant le massacre. Lorsque le texte du projet a été soumis, les États-Unis ont demandé de procéder au vote paragraphe par paragraphe et se sont abstenus sur deux paragraphes préliminaires, notamment le paragraphe six dans lequel le Conseil « Réaffirmait ses résolutions pertinentes qui affirment que la IV Convention de Genève du 12 août 1949 est applicable aux Territoires occupés par Israël en juin 1967, y compris Jérusalem, ainsi que les responsabilités d'Israël à cet égard. » L'ambassadeur des États-Unis, Madeleine Albright, a déclaré que c'était «précisément pour servir et protéger le processus de paix que les États-Unis avaient - à grand regret - pris la difficile décision de permettre que cette résolution soit adoptée. »" D'autres remarques indiquent que la désapprobation des États-Unis porte sur la référence faite dans ces deux paragraphes à la phrase «Territoires palestiniens occupés » et surtout sur la référence à Jérusalem, bien que ce langage ne soit pas nouveau pour le Conseil de sécurité. L'ambassadeur Albright aurait déclaré que :

"Jérusalem était l'une des questions les plus délicates à aborder au cours des négociations. C'était une affaire à régler entre les parties. L'évocation de la question de Jérusalem dans le texte ne pouvait pas porter préjudice ou préjuger du résultat des négociations de paix. Les États-Unis ne pouvaient soutenir aucun effort du Conseil de sécurité pouvant porter préjudice à l'issue du processus de paix au Moyen-Orient".»

21 La position des États-Unis qui consiste à refuser de considérer Jérusalem comme un "territoire occupé» ou d'y reconnaître l'applicabilité du droit international humanitaire a sérieusement préoccupé aussi bien les organisations des droits de l'homme que les milieux politiques palestiniens. Chose inquiétante également: l'incapacité du Conseil de sécurité à réaffirmer l'illégalité des colonies par rapport au droit international, alors que l'attention de la communauté internationale avait été attirée, de manière aussi dramatique, sur la question. Enfin, il est évident que le Conseil n'a pas directement demandé à Israël de s'acquitter des obligations qu'il a contractées aux termes de la IV . Convention de Genève et n'a fait aucune mention non plus des responsabilités des États tiers en la matière. Dans le paragraphe clé de la résolution 904 de 1994, le Conseil demande que : « des mesures soient prises pour garantir la sécurité et la protection des civils palestiniens dans tous les Territoires occupés, y compris, entre autres, une présence internationale ou étrangère temporaire, prévue par la Déclaration de principes (S/26560) et ce, dans le cadre du processus de paix en cours. » 
Lapproche suivie par le Conseil de sécurité - et par l'UE - à la suite du massacre de la mosquée d'Hébron contraste avec ses positions antérieures au processus de paix. Dans les précédentes résolutions où il demandait à Israël de se conformer à la Convention et, plus particulièrement, dans sa résolution 681 de 1990 où il demandait aux autres États de faire respecter ladite convention, le Conseil de sécurité donnait mandat d'entreprendre une action fondée sur le droit en vue d'assurer toute la protection garantie par le droit international humanitaire. Les mesures de surveillance de la situation et de présentation d'un rapport pour lesquelles le Secrétaire général avait été mandaté s'inscrivaient clairement dans ce cadre plus large. Cependant, dans la résolution 904 (1994) du Conseil de sécurité, la question de la participation des parties tierces à la "présence internationale " et, évidemment, les autres "mesures pour garantir la sécurité et la protection» des Palestiniens, semblent avoir été traitées essentiellement comme une assistance offerte aux parties palestinienne et israélienne plutôt que comme un moyen particulier de s'acquitter des obligations légales existantes auxquelles les parties tierces sont tenues en vertu du droit international humanitaire.

Le résultat opérationnel de la résolution 904 (1994) a été le déploiement de la présence internationale temporaire à Hébron pour les trois mois de l'été 1994". Deux États membres de l'UE, le Danemark et l'Italie, se sont joints à la Norvège pour apporter leur contribution en personnel. Les termes de références de la TIPH, tout comme le langage de la résolution 904 de 1994, semblent attribuer un caractère sui generis à l'incident qui leur avait donné lieu et ne pas faire de lien entre la présence internationale et la violation systématique du droit international humanitaire. Le texte final de l'accord sur la sécurité à Hébron prévoyant le déploiement d'une TIPH énonce les tâches de son personnel TIPH comme suit :

- fournir par leur présence un sentiment de sécurité aux Palestiniens d'Hébron ;

- aider à promouvoir la stabilité et l'environnement favorable menant à l'amélioration du bien-être et du développement économique des Palestiniens d'Hébron;

- surveiller les efforts visant à rétablir la sécurité des Palestiniens et les évènements qui

l'affectent, ainsi que la reprise d'une vie normale dans la ville d'Hébron;

- fournir des rapports comme prévu dans le paragraphe (A : 5) ci-dessous.

Un mémorandum d'accord signé par les États européens participants inclut dans ses directives opérationnelles que la «TIPH élaborera des rapports quotidiens sur la situation selon les normes des droits de l'homme... »". Le Mémorandum ne comporte aucune précision relative aux normes à appliquer ou à d'autres questions soulevées par ces directives implicites, bien qu'il prévoie que «les membres de la TIPH n'interviendront pas dans les conflits ou les incidents. Ceux-ci feront l'objet d'un compte rendu présenté immédiatement au Chef de mission. $\aleph^{m}$ La correspondance entretenue par une association de groupes palestiniens des droits de l'homme avec la TIPH tentant d'apporter des éclaircissements sur les critères, les normes et les instruments internationaux spécifiques sur la base desquels la TIPH devait évaluer la situation à Hébron s'est soldée par un échec (Welchman, 1996)". Il était cependant clair que la TIPH n'avait pas à aborder le problème des violations structurelles importantes du droit international, qui sont si flagrantes dans la ville d'Hébron. On peut supposer que des expressions comme « rétablir la sécurité » et « reprise de la vie normale dans la ville d'Hébron » ont pu susciter le mécontentement si, comme il le semble, elles étaient interprétées comme le retour au statu quo qui existait avant le massacre de la mosquée. Bref, le problème conceptuel particulier avec la TIPH est qu'elle risquait, par 
inadvertance, d'aggraver les perceptions populaires d'un désengagement de la part des principales parties tierces par rapport à l'application rigoureuse du droit international humanitaire, $\mathrm{y}$ compris en matière de surveillance et de sécurité véritable.

Le respect des droits de l'homme en politique étrangère : l'accord d'association israéloeuropéen

L'Accord d'association est un exemple plus récent de la manière dont l'UE semble traiter la tension évidente qui existe entre les exigences du processus diplomatique de paix et le droit international, d'une part, et le rôle et les responsabilités incombant aux parties tierces, de l'autre. En 1996, des Parlementaires nationaux dans les États membres de l'UE ont été chargés de ratifier cet accord qui s'inscrit dans la série d'accords euro-méditerranéens avec des États non européens de la région méditerranéenne, ce qui comprend l'Égypte, le Maroc, la Tunisie, Israël et l'accord intérimaire avec l'OLP. Tous ces accords comportent un article 2 standard qui stipule que :

«Les relations entre les parties, de même que toutes les dispositions de l'accord luimême, seront fondées sur le respect des droits de l'homme et les principes démocratiques qui guident leur politique interne et internationale et constituent un élément essentiel du présent accord ».

Les termes «élément essentiel » se retrouvent également dans les accords passés par l'UE avec d'autres États et s'inscrivent dans la question politique plus large des relations extérieures de l'UE. Un autre article prévoit que chacune des parties peut prendre les «mesures appropriées " si elle considère que l'autre partie ne s'est pas acquittée de l'une des obligations qu'elle a contractées en vertu de l'accord. Une attention particulière a été accordée à cet égard aux "cas d'urgence particulière " définis par la Commission européenne comme « une infraction matérielle de l'accord par l'une des parties, [y compris] la violation d'éléments essentiels de l'accord. ${\aleph^{n}}^{\mathrm{L}} \mathrm{La}$ violation systématique des droits de l'homme constitue donc une infraction matérielle à l'accord.

27 L'inclusion de telles clauses dans ces accords d'association qui couvrent les relations commerciales et politiques entre les États concernés, a ajouté au débat sur les conditionnalités qui, en fonction des participants, peut comporter des questions relatives aux motifs, aux perceptions, aux implications, aux méthodes de mise en œuvre et au risque de sélectivité dans l'application. L'accord avec Israël remplace le précédent Accord de coopération de 1975 qui régissait les échanges commerciaux entre l'UE et Israël et n'avait pas la même teneur en matière de droits de l'homme. Au cours des négociations qui ont duré plusieurs années, l'UE a clairement précisé sur plusieurs points que la mise à jour et le développement de ses relations avec Israël dépendaient des progrès réalisés dans le processus de paix.

Pour les organisations des droits de l'homme, les agences de développement et d'autres observateurs de la politique de l'UE dans la région, l'article 2 offrait certaines possibilités à un moment où la région connaissait de fortes tensions et où les sérieuses violations en cours suscitaient des déclarations réprobatrices. Mais, vu de l'extérieur, l'article ne contenait rien de substantiel pouvant donner lieu à des mesures coercitives. Le contexte particulier de 1996-97 était celui de la poursuite des activités d'implantation israéliennes, de la torture et du mauvais traitement, officiellement condamnés, infligés aux détenus palestiniens", des politiques discriminatoires appliquées contre les habitants palestiniens de Jérusalem" et du bouclage prolongé et 
punitif de la Cisjordanie et de la bande de Gaza". Les organisations des droits de l'homme" ont fait remarquer, aussi bien sur le plan local qu'international, que certaines questions étaient d'ordre politique et devaient, à ce titre, être étudiées par les gouvernements de l'UE qui avaient l'intention de conclure un accord avec le gouvernement d'Israël, lequel en effet affirmait que sa politique intérieure et internationale était " guidée par le respect des droits de l'homme.»

Par ailleurs, durant le processus de ratification, des Parlementaires de certains pays européens ont demandé à leurs gouvernements des éclaircissements sur le sens et les implications des clauses relatives aux droits de l'homme. Entre autre, ils voulaient que soit consigné dans le procès-verbal que l'expression "respect des droits de l'homme " inclut les termes de droit international humanitaire, quand applicables, ainsi que les instruments du droit international humanitaire qui lie les parties.

De leur côté, certains gouvernements des pays membres de l'UE semblaient ralentir le processus de soumission de l'accord à l'assemblée parlementaire de leur pays pour ratification en raison du mécontentement croissant vers la fin de 1996 suscité par la conduite du gouvernement Netanyâhû dans le processus de paix. Au début du sommet présidentiel tenu en octobre 1996 à la suite des troubles et des pertes humaines provoqués, dans les Territoires occupés, par l'ouverture d'un accès à un tunnel à proximité de la mosquée al-Aqsâ à Jérusalem-Est, le Conseil européen a réaffirmé ses positions sur l'inadmissibilité de l'acquisition de territoire par la force et sur l'applicabilité de la $\mathrm{IV}$. Convention de Genève à Jérusalem-Est comme à tous les autres Territoires occupés. La déclaration poursuit en ces termes :

"Le Conseil souligne l'importance de l'Accord d'association euro-méditerranéen qui est fondé sur l'engagement commun au processus de paix. Dans ce contexte, il demande à Israël de démontrer clairement dans la pratique son intention de respecter intégralement les accords déjà conclus avec l'OLP $\aleph^{\prime}$.

31 À la fin de l'année, le Conseil européen prête une attention particulière aux activités d'implantation déclarant que : « La question des implantations a pour effet de miner la confiance dans le processus de paix. Les implantations sont une infraction au droit international et constituent un obstacle majeur à la paix. »Cette déclaration est suivie d'une nouvelle mention de l'accord d'association :

«Le Conseil européen rappelle que les accords d'association avec l'Autorité

palestinienne et avec Israël engagent les parties à promouvoir la conformité aux

normes fondamentales de la démocratie, y compris le respect des droits de l'homme et de l'État de droit $\%$.

Le Conseil a donc lui-même fait référence au cours des derniers mois de l'automne 1996 aux deux éléments qu'il souhaitait promouvoir par le biais de l'accord d'association : l'avancement $\mathrm{du}$ processus de paix, qui était et a toujours été une exigence contextuelle, ainsi que le « respect des droits de l'homme et de l'État de droit » qui, en vertu des termes de l'article 2 commun à tous les accords d'association, constituent une exigence textuelle. Il a en outre réaffirmé ses positions sur l'applicabilité du droit international humanitaire à deux questions d'une importance capitale : les colonies et Jérusalem. L'argument selon lequel la mise en œuvre de l'État de droit augmente les chances de promouvoir un processus de paix viable a été, comme on l'a vu plus haut, explicitement reconnu dans les années précédentes. Néanmoins, étant donné le contexte du processus de paix, de fortes tensions devaient apparaître. 
33 En janvier 1997, la signature du Protocole d'Hébron", si longtemps attendu, a été accueillie avec grand soulagement par l'UE et d'autres États membres de la communauté internationale. En soumettant au Parlement britannique l'accord d'association euro-méditerranéen pour ratification, Malcom Rifkind, ministre britannique des Affaires étrangères de l'époque, a déclaré que : « le Protocole d'Hébron qui a été conclu montre qu'Israéliens et Palestiniens soutiennent la poursuite du processus de paix. Je ne vois par conséquent aucune raison de reporter la ratification de l'Accord euro-israélien. J'estime qu'il devrait être mis en œuvre le plus tôt possible. $»^{\star \prime}$.

Le gouvernement d'Israël ayant satisfait à l'exigence contextuelle d'avancer dans le processus de paix, le gouvernement britannique (de même que d'autres États membres de l'UE) avaient hâte de faire approuver l'accord par le Parlement". Durant les débats parlementaires qui se sont déroulés cette année sur la ratification, des éclaircissements utiles et intéressants ont été apportés en réponse aux questions sur le sens et les implications de l'expression « respect des droits de l'homme » dans l'article 2 de l'accord ${ }^{\text {. }}$ Toutefois, jusqu'à présent, il n'avait jamais été mentionné dans les comptes rendus que cela incluait les dispositions du droit international humanitaire, lorsqu'elles s'appliquent, et que, par conséquent, des violations comme les activités d'implantation et autres mesures d'annexion auraient les mêmes conséquences sur la mise en vigueur de l'accord, tout comme les autres «cas d'urgence particulière » reconnus comme infraction matérielle à l'accord. L'accord d'association précise également la position de l'UE sur le statut légal de Jérusalem et des colonies en incluant des clauses standard relatives à son applicabilités. Au Royaume-Uni, les parlementaires ont exprimé leurs profondes inquiétudes quant à l'entrée sur les marchés de marchandises produites dans les colonies de peuplement sous la mention " produites en Israël » afin de répondre aux conditions préférentielles prévues par les accords commerciaux entre Israël et l'Europe“. En réponse à l'un des membres du Parlement, le gouvernement de l'époque a traité la question de la manière suivante :

«La position du gouvernement britannique concernant Jérusalem est bien connue de tous nos partenaires européens... Nous ne reconnaissons pas la souveraineté (de jure) d'Israël sur n'importe quelle partie de Jérusalem. Il s'ensuit que les marchandises produites à Jérusalem ne sont pas des produits israéliens. La même logique s'appliquerait aux marchandises produites dans les colonies israéliennes des Territoires occupés »".

Que l'UE parvienne à obtenir du gouvernement d'Israël des garanties en la matière, qui correspondent aux positions adoptées de longue date sur le respect du cadre juridique international, a toujours dépendu de leur volonté politique d'insister sur le contexte de leurs propres relations commerciales et politiques. Cependant, de manière générale, les discussions relatives à la clause des droits de l'homme dans l'accord d'association euroisraélien sont révélatrices des choix faits par les États membres de l'UE dans le processus de paix. Maintenant leurs «positions de principe " sur l'applicabilité intégrale du droit international humanitaire, ils ont néanmoins limité entre-temps tout effort de mise en œuvre de l'État de droit aux dynamiques du processus de paix qui, tel qu'il a été conçu, ne prévoit pas l'application du droit.

Le refus de parler de mise en vigueur : la conférence de dix minutes

Les perturbations chroniques du processus de paix suscitées par de sérieuses violations du droit international humanitaire ont eu pour effet de retarder dans l'agenda des co- 
partenaires d'Israël à la convention les perspectives de mise en œuvre. La décision particulièrement provocatrice prise par le gouvernement Natanyâhû de construire une colonie de peuplement Har Homa à Jabal Abû Ghunaym, à Jérusalem, les mesures déniant aux Palestiniens le droit de résider à Jérusalem ainsi que les autres violations permanentes ont conduit, au printemps 1997, à la convocation de la dixième session extraordinaire d'urgence de l'Assemblée générale des Nations unies". Étant donné la menace qui pesait sur la paix internationale en raison des mesures illégales prises par Israël et l'impossibilité d'entreprendre une action à travers le Conseil de sécurité à cause du veto des États-Unis, cette session a eu lieu dans le cadre de l'Union pour le maintien de la paix. Dans la série de résolutions" adoptées au cours de la session, l'Assemblée générale a demandé à Israël d'admettre l'applicabilité de jure de la Convention dans les territoires qu'il occupe depuis 1967 et de revenir sur toutes les mesures qui enfreignent la Convention (particulièrement celles qui concernent Jérusalem). Elle a, en outre, demandé aux hautes parties contractantes de "convoquer une conférence sur les mesures de mise en vigueur de la Convention dans les Territoires palestiniens occupés, y compris Jérusalem, et d'assurer le respect de ladite convention conformément à l'article 1 ». L'Assemblée générale s'est par ailleurs réservé le droit de "reconsidérer la situation en vue de faire de nouvelles recommandations aux États membres des Nations unies" si Israël continuait à menacer la paix internationale et la sécurité par ses infractions à la Convention.

Dans les résolutions successives de la session extraordinaire d'urgence, l'Assemblée générale des Nations unies a recommandé que la Suisse, en tant que gardienne de la Convention, prenne les mesures nécessaires pour la convocation de la conférence, y compris la convocation d'une réunion d'experts. Le gouvernement suisse a entrepris, avec un manque d'enthousiasme évident, une tournée de consultation auprès des hautes parties contractantes à la suite de laquelle il a déclaré n'avoir pu aboutir à un consensus sur l'opportunité de réunir la conférence recommandée par l'Assemblée générale. La Suisse a, par la suite, proposé sa propre approche «à deux voies » et a organisé - comme premier pas - et accueilli une réunion quadripartite à huis clos en juin 1998, regroupant les représentants de l'OLP et d'Israël en présence du Comité international de la Croix Rouge. Cette mesure a été considérée par plusieurs observateurs comme parfaitement inappropriée, dès lors qu'elle risquait de rejeter la charge d'assurer le respect de la Convention par une puissance occupante récalcitrante - charge qui incombait aux hautes parties contractantes - sur le représentant des personnes protégées". Durant la réunion, les parties n'ont pas réussi à s'entendre sur la faisabilité et l'opportunité d'un dialogue quadripartite dans le contexte du refus permanent d'Israël d'admettre l'applicabilité de jure de la Convention et de cesser les violations en cours.

En octobre 1998, la Suisse, en tant que gardienne de la Convention, a convoqué une réunion d'experts pour discuter des "problèmes généraux concernant la IV Convention de Genève en général et plus particulièrement en Territoire occupé. » Aussi bien les États-Unis qu'Israël ont accepté la convocation d'une telle réunion à condition que les termes de référence ne comportent pas la discussion du cas particulier des Territoires palestiniens occupés. Ceci révèle malheureusement l'écart manifeste entre la volonté exprimée dans les résolutions de l'Assemblée générale et les actions menées en conséquence. La Suisse ne s'est pas risquée à convoquer la réunion souhaitée par la grande majorité des hautes parties contractantes dès lors que n'importe quel État partie s'y était opposé. Concernant la conférence générale officielle que l'OLP et un 
grand nombre de hautes parties contractantes l'avaient pressé de convoquer, la Suisse a continué à invoquer l'absence de consensus entre les hautes parties contractantes.

En février 1999, l'Assemblée générale des Nations unies s'est de nouveau réunie en session extraordinaire d'urgence et a réitéré sa recommandation concernant la réunion des hautes parties contractantes en vue d'examiner les mesures à prendre pour mettre en vigueur la Convention dans les Territoires palestiniens occupés. Cette conférence devait se tenir le 15 juillet. À ce moment-là, devant l'intransigeance du gouvernement Natanyâhû en ce qui concerne aussi bien la Convention que le processus de paix, les principaux États européens et non européens ont trouvé qu'ils avaient de bonnes raisons de prendre les mesures nécessaires pour faire respecter le droit et ce, en dépit des protestations des États-Unis et d'Israël. La Suisse a répondu par une nouvelle tournée de consultation. En mai 1999, Ehud Barak a été élu Premier ministre d'Israël et l'image politique a à nouveau changé. Un nombre limité mais croissant d'États influents a alors commencé à plaider en faveur du report de la conférence, reprenant l'argument soutenu précédemment: le manque de cohérence entre la mise en œuvre du droit et l'espoir d'une relance du processus de paix par Barak. Les États-Unis" et Israël se trouvaient à la tête des pays exerçant une pression considérable sur les États européens qui voulaient maintenant attendre de voir quelles étaient les intentions de Barak. Les contre-arguments étaient que Barak ne devait pas être récompensé d'avance par le report de la conférence alors qu'il n'avait donné aucun signe clair ni pris aucun engagement sérieux quant à l'abandon des politiques de longue date qui, à la fois, violent la Convention et interrompent le processus de paix, au point de l'arrêter complètement sous le gouvernement de Natanyâhû.

Pendant que les contacts diplomatiques intensifs se poursuivaient, les organisations palestiniennes et internationales des droits de l'homme ont demandé aux hautes parties contractantes d'honorer leurs engagements et d'éviter la politisation du droit". L'Australie et le Canada se sont joints aux États-Unis et à Israël en annonçant qu'ils ne participeraient pas à la conférence ; avec un regret évident, l'UE a changé de position. Pour finir, cent trois des cent quatre-vingt-huit États parties à la Convention ont participé à la conférence, qui a duré dix minutes, et ont publié une brève déclaration annonçant que la conférence s'était tenue à Genève conformément à la résolution ES-10/6, réaffirmant l'application de la IV convention de Genève aux Territoires palestiniens occupés, y compris Jérusalem, réitérant la «nécessité du respect intégral des dispositions de ladite Convention dans ce territoire » et concluant :

«Prenant en considération l'amélioration de l'atmosphère au Moyen-Orient dans

l'ensemble, la conférence a été ajournée étant entendu qu'elle se réunira de nouveau à

la lumière des consultations relatives au développement de la situation humanitaire sur le terrain. »

41 L'« atmosphère améliorée » s'est avérée fugace et, deux ans plus tard, les hautes parties contractantes ont à nouveau dû, à la lumière de la seconde intifâda, convoquer une conférence.

La seconde intifâda : appels renouvelés pour une protection internationale

La seconde intifâda a éclaté en septembre 2000 à la suite de la visite très provocatrice d'Ariel Sharon, alors chef du Likoud et bientôt Premier ministre d'Israël, au Haram alSharîf (esplanade des Mosquées), qui se situe dans Jérusalem-Est sous occupation. Le nombre croissant de morts et de blessés parmi la population civile palestinienne a suscité des appels à la protection internationale faisant écho à ceux qui avaient été 
lancés durant la première intifâda. L'opposition d'Israël à ces appels devait, par la suite, entraîner le veto des États-Unis au Conseil de sécurité. En octobre, les États-Unis se sont abstenus lors du vote de la résolution 1322 du Conseil de sécurité qui, entre autres, déplorait l'acte de provocation commis au Haram al-Sharîf à Jérusalem, condamnait les actes de violence, particulièrement le recours excessif à la force contre les Palestiniens, et demandait à Israël de « se conformer scrupuleusement à ses obligations juridiques et aux responsabilités qui lui incombent en vertu de la IV Convention de Genève. » Le même mois, une session spéciale de la Commission des Nations unies aux droits de l'homme a largement adopté la résolution S-5/1 déclarant qu'elle était " profondément préoccupée par l'ampleur et la gravité des violations systématiques des droits de l'homme commises par Israël, puissance occupante, et qui sont des crimes de guerre, des violations flagrantes du droit international humanitaire et des crimes contre l'humanité » et a établi d'urgence une commission d'enquête sur les droits de l'homme. Formée de trois personnes, cette commission s'est rendue dans les Territoires palestiniens en février 2001 et a présenté son rapport en mars. Au titre de ses multiples recommandations, il est dit: "Une présence internationale doit être établie pour surveiller et rendre compte régulièrement de la conformité de toutes les parties aux normes des droits de l'homme et du droit humanitaire, de sorte à assurer à la population des Territoires occupés l'entière protection des droits de l'homme. Une telle présence internationale devrait être immédiatement établie et constituée de manière à refléter le sentiment d'urgence au sujet de la protection des droits de l'homme du peuple palestinien $"$.

43 La Commission d'enquête sur les droits de l'homme a affirmé que la nécessité d'une telle protection était strictement conforme à la IV. Convention de Genève et que «les hautes parties contractantes, devaient, individuellement et collectivement, entreprendre une action appropriée et efficace pour faire face à une situation d'urgence qui demande que des mesures soient prises pour alléger les souffrances quotidiennes résultant des graves infractions de la Convention.» Le rapport constate que les hautes parties contractantes "devraient agir d'urgence pour convoquer à nouveau la Conférence» qui, cette fois, "devrait mettre en place un mécanisme international efficace pour prendre les mesures d'urgence nécessaires. $»^{\star}$ À la suite de la présentation du rapport, de longues négociations ont eu lieu au Conseil de sécurité concernant les projets de résolutions mettant l'accent sur les mécanismes de protection, mais, finalement, le projet a été présenté, dans sa forme originale, par les membres non alignés de la commission". Le texte comportait une clause précisant que le Conseil était disposé à agir sur mandat du Secrétaire général « pour mettre en place un mécanisme approprié assurant la protection des civils palestiniens, y compris par la constitution d'un corps d'observateurs. " Les États-Unis ont opposé leur veto à la résolution et les États membres de l'Union européenne se sont abstenus. Par la suite, dans son rapport d'octobre 2001, le Rapporteur spécial de la Commission des droits de l'homme sur la situation des droits de l'homme dans les Territoires palestiniens occupés par Israël depuis 1967 a de nouveau signalé que « le temps est venu de prévoir une présence internationale dans la région qui serait chargée de surveiller et de réduire l'usage de la violence. Cette décision qui s'impose est celle retenue par le G-8 des ministres des Affaires étrangères lors de leur réunion à Rome les 18 et 19 juillet 2001". Le Rapporteur spécial a de la peine à comprendre pourquoi la communauté internationale n'a pas sérieusement essayé de persuader Israël d'accepter une présence de cette nature (l'Autorité palestinienne l'ayant déjà acceptée). Des missions 
internationales d'observation ou de maintien de la paix ont été envoyées à travers le monde dans des situations beaucoup moins explosives et il n'y a aucune de raison pour qu'il n'en soit pas de même dans les Territoires palestiniens occupés "".

Après les attaques terroristes du 11 septembre aux États-Unis, le premier ministre israélien Ariel Sharon a cherché à assimiler la position d'Israël vis-à-vis des Palestiniens à celle des États-Unis dans leur "guerre contre le terrorisme.» La situation dans les Territoires occupés et en Israël continuant à se détériorer, la conférence des hautes parties contractantes s'est de nouveau réunie le 5 décembre 2001. Il ne s'agissait pas de débats mais plutôt de faire une déclaration déjà préparée et approuvée. La réunion n'a donc pas servi de forum à des discussions substantielles sur les mécanismes d'application, de mise en œuvre ou de protection. Néanmoins, elle évoquait les obligations des parties tierces aux termes du droit international humanitaire et faisait référence à l'article 1 en insistant, évidemment, sur les infractions graves et en réaffirmant l'applicabilité de la $\mathrm{IV}$ Convention de Genève en Territoires palestiniens occupés, y compris Jérusalem. Dans leur déclaration, les cent quatorze États présents à cette réunion se sont dits favorables à «tous les règlements et tous les accords que soutiendraient les parties au conflit concernant le déploiement d'observateurs indépendants et impartiaux pour surveiller, entre autres, les infractions à la IV o Convention en tant que mesure de protection et de restauration de la confiance afin d'assurer l'efficacité des règles humanitaires. »Cela ne tranche évidemment pas la question des efforts à déployer pour amener Israël à accepter ce déploiement. La déclaration reconnaît également l'utilité du droit international dans l'instauration de la paix, mettant l'accent sur le fait que «le respect de la IV Convention et du droit international humanitaire en général est essentiel pour améliorer les conditions humanitaires sur le terrain et pour instaurer une paix juste et durable. » Les États-Unis n'ont pas participé à cette conférence et le porte-parole de l'Administration américaine l'aurait qualifiée de "contre-productive ». La réunion de Genève fut suivie d'un autre veto des États-Unis au Conseil de sécurité. Manifestant sa continuelle frustration par rapport au Conseil de sécurité, la 10 Session extraordinaire d'urgence de l'Assemblée générale s'est à nouveau réunie, a adopté une résolution confirmant et avalisant la Déclaration de Genève et a demandé que cette Déclaration soit respectée par toutes les hautes parties contractantes, tous les membres des Nations unies, les observateurs et les agences.

Les évènements tragiques et violents de l'année suivante ont encore plus mis en évidence la nécessité de donner à ces engagements davantage de consistance de la part des États tiers les plus puissants et ce, en redoublant leurs efforts politiques visant à désamorcer la montée en spirale de la violence, aussi bien en Palestine qu'en Israël, et en s'attachant tout particulièrement au droit humanitaire. L'année 2002 a vu le redéploiement des troupes et des chars israéliens dans les plus grands centres de Cisjordanie et de Gaza, y compris le blocus des quartiers généraux de Arafât à Ramallah, avec les couvre-feux prolongés, les restrictions sévères imposées à la liberté de circulation et les attaques au missile, ainsi que le refus d'Israël de coopérer avec l'équipe d'établissement des faits, relevant des Nations unies sur mandat du Secrétaire général", qui était chargée de recueillir des informations exactes sur les évènements survenus en avril dans le camp de réfugiés de Jénine. 2002 a aussi connu plus d'attentats suicides commis par des Palestiniens contre des civils à l'intérieur d'Israël et les gouvernements américain et britannique ont donné la priorité aux demandes concernant la réforme de l'Autorité palestinienne. Vers la fin de l'année, la «crise 
humanitaire » vécue par la population civile palestinienne en Cisjordanie et à Gaza était devenue un sujet de préoccupation internationale manifeste. En même temps, alors qu'une autre attaque importante contre l'Irâq se préparait, le gouvernement britannique s'est de nouveau trouvé confronté aux comparaisons entre le traitement réservé par le Conseil de sécurité à la question du non-respect du droit humanitaire par l'Irâq et par Israël, ainsi qu'aux accusations de sélectivité dans sa volonté politique de mettre en vigueur le droit international.

$\mathrm{Au}$ cours des négociations et de la (non-) application des accords d'Oslo, les parties tierces les plus puissantes n'ont pas réussi à combiner les impératifs politiques d'appui $\mathrm{du}$ processus de paix israélo-palestinien et les impératifs juridiques du droit international humanitaire. Le prix de cette négligence a été énorme sur tous les fronts, aussi bien personnels que politiques. D'un point de vue pratique, le refus d'imposer à Israël le respect du droit international humanitaire et cela, par considération pour les exigences politiques d'un processus de paix qui reste encore tributaire des violations de ce droit durant la période "intérimaire » ou de "transition », n'a manifestement pas réussi à produire et à assurer les conditions nécessaires à une solution durable du conflit par les négociations envisagées sur le statut définitif. On peut se demander si, lorsque l'attention sera à nouveau centrée sur le conflit israélo-palestinien, le rôle du droit dans la mise en place et le maintien des conditions d'un règlement pacifique sera aussi systématiquement ignoré.

\section{BIBLIOGRAPHIE}

Al-Haq, 1991, Protection Denied, Continuing Human Rights Violations in the Occupied Palestinian Territories, 1990, Ramallah, 1991.

Azzam F., 1997, « The Duty of Third States to Implement and Enforce International Humanitarian Law », Nordic Journal of International Law.

Pictet J., éd., 1958, Commentaire: IV Convention de Genève relative à la protection des personnes civiles en temps de guerre, Comité international de la Croix Rouge, Genève.

Roberts A., 1992, « Prolonged Military Occupation : The Israeli-Occupied Territories 1967-1988 », dans Playfair (ed.), International Law and the Administration of Occupied Territories, Oxford.

Shammas C., 1999, « Conference of Contention », Middle East International, du 2 juillet.

Shehadeh R., 1985, Occupier's Law : Israel and the West Bank, revised edition, Washington.

Stephens M., 1989, Enforcement of International Law in the Israeli-Occupied Territories, Al-Haqq, Ramallah.

Welchman L., 1996, “International Protection and International Diplomacy”, dans CIHRE/Pax Christi, International Human Rights Enforcement: The Case of the Occupied Palestinian Territories in the Transitional Period, Ramallah, 1996. 


\section{NOTES}

1.Accord intérimaire israélo-palestinien sur la Cisjordanie et la bande de Gaza, conclu à Washington le 28 septembre 1995.

2.IV ${ }^{\text {e }}$ Convention de Genève relative à la protection des personnes civiles en temps de guerre, du 12 août 1949.

3.Article 1 commun aux quatre conventions de 1949.

4.Dans une lettre adressée à deux membres du Parlement britannique, le Comité international de la Croix Rouge exprime sa position en ces termes : « Le Comité international de la Croix Rouge considère vital que les États parties aux conventions de Genève prennent toutes les mesures possibles pour assurer le respect de ce corpus de droit, dans le but de diminuer les souffrances des personnes touchées par le conflit armé. En outre, ces États ont l'obligation morale de le faire parce que, en tant que parties aux conventions de Genève, ils se sont engagés, non seulement à respecter lesdites conventions, mais aussi à les faire respecter par les autres États en toutes circonstances. Tel est la teneur de l'article 1 commun aux quatre conventions ». Lettre de Yves Sandoz (Director, Principles, Law and Relations with the Movement, ICRC) aux Parlementaires Ernie Ross et Sir Dennis Walters, reproduite dans Towards a Strategy for the Enforcement of Human Rights in the Israeli Occupied West Bank and Gaza : A Working Symposium 25 July 1989, Labour and Conservative Middle East Councils, Londres, p. 7-8. 5.Article 146 de la IV ${ }^{\mathrm{e}}$ Convention (article 49 dans la I ${ }^{\mathrm{e}}$ Convention, 50 dans la II ${ }^{\mathrm{e}}$ et 129 dans la III $)$.

6.Article 47 de la Convention ; Pictet, 1958, p. 272-276.

7.Lors de sa première occupation de la Cisjordanie, Israël avait en fait reconnu l'applicabilité de la Convention aux Territoires occupés. L'article 35 de la Proclamation militaire $\mathrm{N}^{\circ} 3(7 / 6 / 67)$ exige que le tribunal militaire et les officiers « appliquent les dispositions des Conventions de Genève du 13 août 1949 relatives à la protection des personnes civiles en temps de guerre pour ce qui se rapporte aux mesures légales. En cas de contradiction entre les ordres et les dispositions de la Convention de Genève, les dispositions de cette dernière s'appliquent. » Cet article a été supprimé par l'Ordre militaire 144 d'octobre 1967. Voir Raja Shehadeh, 1985. Depuis ce moment, la position officielle d'Israël est que la Convention ne s'applique pas de jure aux Territoires occupés en 1967, mais qu'Israël suit les « dispositions humanitaires » de facto. La Convention elle-même ne fait pas de distinction entre « humanitaire » et toute autre disposition. Israël ayant ratifié la IV ${ }^{e}$ Convention de La Haye relative au respect des normes découlant du droit coutumier en temps de guerre signée à Londres le 18 octobre 1907, ainsi que ses règlements annexes, les obligations qui en découlent s'appliquent à son comportement dans les Territoires occupés. Voir Roberts, 1992, sur ce qu'il appelle « une petite confusion logique peu remarquée sur l'applicabilité des conventions de La Haye et Genève ».

8.En 1972, Israël a rejeté l'offre officielle du Comité international de la Croix Rouge d'agir en tant que délégué officiel de la puissance protectrice dans ses fonctions humanitaires, conformément à l'article 11 du Rapport annuel du Comité international de la Croix Rouge pour 1972, p. 69-70. Pour sa part, le Comité international de la Croix Rouge a bien précisé que : « les fonctions d'observation relevant des puissances protectrices ou de leurs représentants ou délégués aux termes des Conventions de Genève n'ont pas été confiées au Comité international de la Croix Rouge pour ce qui est du conflit au Moyen-Orient. »International Review of the Red Cross, volume 10, 1970, p. 
429. Pour une plus ample discussion, voir al-Haq, 1990, p. 647. Pour le rôle du Comité international de la Croix Rouge dans les Territoires palestiniens occupés, voir Welchman, 1996, p. 264-265.

9.L'article 5 (3) de la Déclaration de principes (13 septembre 1993) précise que « les questions restant à traiter dans le cadre des négociations sur le statut définitif comportent : Jérusalem, les réfugiés, les dispositions relatives à la sécurité, les frontières, les relations et la coopération avec les autres pays voisins, ainsi que d'autres thèmes d'intérêt commun ".

10.Pour une analyse complète de la résolution 242 (1967) du Conseil de sécurité et des différents débats qui ont tourné autour, voir Musa Mazzawi, 1997.

11.Pour une analyse détaillée des différentes phases et des accords relatifs au processus de paix israélo-palestinienne, voir Shehadeh, 1985.

12. Rapport soumis au Conseil de sécurité par le Secrétaire général conformément à la résolution 605 (1987) : UN Doc.S/194433 du 21 janvier 1988, para. 27.

13.Par exemple, la déclaration UN Doc.S/20156 du 25 août 1988 à la suite de l'émission par les autorités israéliennes de 25 ordres de déportation en un jour et, en juin 1990 la déclaration UN Doc.S/21369 du 19 juin 1990 à la suite du veto des États-Unis sur la « résolution de protection ». Voir Welchman, 1996, article duquel certains passages de cet exposé sont tirés.

14.L'Assemblée générale a invoqué l'article I plus fréquemment (Azzam, 1997, p. 62-63). De son côté, le Conseil de sécurité le mentionne directement de temps à autre comme dans la résolution 469 (1981) du Conseil de sécurité « rappelant la IV Convention de Genève de 1949 et en particulier l'article I ». (Stephens, 1989, p. 13-14).

15.UN Doc.S/21326, du 31 mai 1990.

16.Une partie de cet aperçu des développements de la politique des États tiers au cours de 1990 a été publiée pour la première fois dans Al-Haq, 1991, p. 194.

17.Déclaration du Conseil européen sur le Moyen-Orient, Dublin, 26 et 27 juin 1990. 18. Résolutions 670 (1990) et 674 (1990).

19.Selon l'article 147 de la IV Convention de Genève, « les infractions graves visées à l'article précédent sont celles qui comportent l'un ou l'autre des actes suivants, s'ils sont commis contre des personnes ou des biens protégés par la Convention : l'homicide intentionnel, la torture ou les traitements inhumains, y compris les expériences biologiques, le fait de causer intentionnellement de grandes souffrances ou de porter des atteintes graves à l'intégrité physique ou à la santé, la déportation ou le transfert illégaux, la détention illégale, le fait de contraindre une personne protégée à servir dans les forces armées de la puissance ennemie, ou celui de la priver de son droit d'être jugée régulièrement et impartialement selon les prescriptions de la présente Convention, la prise d'otages, la destruction et l'appropriation de biens, non justifiées par des nécessités militaires et exécutées sur une grande échelle de façon illicite et arbitraire. » Concernant le bilan de l'application de cette définition aux politiques particulières durant l'occupation israélienne de la Cisjordanie et de la bande de Gaza, voir al-Haq, 1991.

20.En décembre 1990, ont été publiées des réponses écrites à des questions ayant rapport au Moyen-Orient adressées au ministre des Affaires étrangères, qui avaient été déposées au Parlement britannique : Question : Demander au Secrétaire d'État aux Affaires étrangères et aux Affaires du Commonwealth s'il a l'intention de proposer au Conseil de sécurité des Nations unies un projet de résolution rappelant à tous les États parties aux Conventions de Genève leur obligation légale d'engager des poursuites dans 
le cas d'un commencement de preuve d'infractions graves aux Conventions de Genève perpétrées par des individus de n'importe quelle nationalité. Réponse : Les États parties aux Conventions de Genève sont bien conscients de leurs obligations légales puisqu'ils sont tenus de s'assurer que les infractions graves à ces Conventions constituent une infraction en vertu de leur législation nationale. Question : Demander au Secrétaire d'État aux Affaires étrangères et aux Affaires du Commonwealth s'il a l'intention de proposer au Conseil de sécurité des Nations unies un projet de résolution rappelant à tous les États engagés dans l'occupation armée les implications des infractions graves aux Conventions de Genève, en ce qui concerne en particulier les traitements réservés par les États à la population civile soumise à l'occupation. Réponse : Les obligations des États parties à la IVe Convention de Genève ont été réitérées par le Conseil de sécurité dans sa résolution 674 à propos de l'occupation iraquienne du Kowît. Hansard Written Answers 20/12/90 c.280.

21.UN Doc.S/22472 du 9 avril 1991.

22.Réponse commune des douze États membres de la Communauté européenne à la lettre du Secrétaire général en date du 6 mars 1991 relative à la mise en œuvre du paragraphe 6 de la résolution 681 ; New York, 15/5/91.

23.Déclaration de Hans Van den Broek, ministre néerlandais des Affaires étrangères, à titre de Président du Conseil des ministres de la Communauté européenne, à la Conférence de Madrid sur le processus de paix au Moyen-Orient, le 30 octobre 1991.

24.Voir Palestinian Human Rights Information Center, The Massacre in Al-Haram alIbrâhîmî al-Sharîf, Context and Aftermath, Jérusalem, 1994.

25.Déclaration du Conseil de l'UE sur le processus de paix au Moyen-Orient, Bruxelles, le $8 / 3 / 94$.

26.UN Press Release SC/5808 du 18 mars 1994, où les Nations unies expriment leur horreur devant « l'effroyable massacre » d'Hébron.

27.Ibid.

28.Un nombre limité d'observateurs est revenu par la suite.

29.Mémorandum d'accord de Copenhague, 2 mai 1994 ; section B : 2.

30.Ibid, section B : 7 .

31.Concernant le débat intégral sur les termes de référence de la TIPH et leur comparaison avec d'autres opérations multilatérales et internationales, voir Welchman, 1996.

32.Sur l'inclusion du respect des principes démocratiques et des droits de l'homme dans les accords entre la Communauté européenne et les pays tiers, voir le Communiqué de la Commission européenne, 23/45/[COM(95) 216 final], p. 13.

33. Voir Amnesty International Report 1997, London, 1997, p. 192-193 : La torture et le mauvais traitement ont continué d'être officiellement et systématiquement cautionnés dans les directives secrètes autorisant le Service de la sécurité publique à faire usage d'une pression physique et morale « modérée ». Le comité ministériel chargé de surveiller le Service de la sécurité publique a continué à accorder trois mois de dérogation pendant lesquels l'usage d'une « pression physique accrue » était autorisé, expression dont le sens reste secret. Les secousses violentes (titul) étaient également admises, mais avec l'autorisation du chef du Service de sécurité. [...] Certaines décisions de la Haute Cour ont permis l'usage de la force physique contre les détenus suspectés d'avoir des informations sur les attaques armées.

34. Voir Living in Jerusalem : An Assessment of Planning Policy, Housing and Living Conditions in the Light of the Palestinians' Right to Adequate Housing, Rapport présenté par Palestinian 
Housing Rights Movement et Habitat International au Comité des Nations unies pour les droits économiques, sociaux et culturels, mai 1996 ; Palestinian Human Rights in Jerusalem, Rapport préparé par les avocats des droits de l'homme en Palestine, juin 1997.

35.Voir Human Rights Watch/Middle East, July 1996, Israel's Closure of the West Bank and Gaza Strip.

36.Par exemple: Memorandum of the Centre for International Human Rights Enforcement of 17/2/97 sur le respect des droits de l'homme dans l'Accord d'association israélo-européen ; la lettre datée du 13 mars 1997 adressée par le Center for Economic and Social Rights au ministre irlandais des Affaires étrangères ; le communiqué de presse du 22 janvier 1997, Human Rights Watch : « Human Rights Watch engage le gouvernement britannique à ratifier l'Accord d'association euroméditerranéen pour assurer une meilleure observation des droits de l'homme en Israël. »

37.Déclaration du Conseil européen sur le processus de paix au Moyen-Orient $\mathrm{du}$ 1/10/96. Cette déclaration intervient à la suite de la résolution 1073 (1996) du Conseil de sécurité en date du 28 septembre 1996, qui n'a pas réussi à mentionner la IV. Convention de Genève, mais a demandé « la cessation immédiate de tous les actes qui ont eu pour résultat d'aggraver la situation et qui ont des effets négatifs sur le processus de paix du Moyen-Orient »; " que la sécurité et la protection de la population civile palestinienne soient assurées »; « que les négociations reprennent immédiatement sur la base convenue dans le cadre du processus de paix du MoyenOrient et que les accords conclus soient appliqués dans les délais prévus. » 38.Déclaration du Conseil européen sur le processus de paix au Moyen-Orient, 14/12/96, Dublin.

39.Protocole relatif au redéploiement à Hébron, 15 janvier 1997.

40.Hansard Oral Answers, 15 janvier 1997, c.319.

41.Les deux chambres du Parlement ont donné leur consentement le 19 février 1997 ; le processus de ratification s'est achevé le 8 avril 1997. À la fin de la même année, la France et la Belgique étaient les seuls pays membres de l'UE à ne pas avoir achevé le processus de ratification au niveau national.

42.Voir Submission to the (UK) Foreign Affairs Committee, Novembre 1997.

43. L'Article 38 de l'accord intérimaire euro-israélien sur le commerce et les affaires liées au commerce qui met en œuvre essentiellement les dispositions de l'accord d'associations relatives au commerce en attendant l'entrée en vigueur de ce dernier et l'article 83 de l'accord d'association euro-israélien.

44.Réponses écrites de Hansard du 18 février 1997 : Question (M. Gunnell) : Demander au Secrétaire d'État aux Affaires étrangères et du Commonwealth quelle est la position qui est prise au sujet de la conformité d'Israël aux articles I et 38 de l'accord intérimaire israélo-européen relatif au commerce et aux questions liées au commerce. Réponse (M. Hanley) : L'article I concerne le respect des droits de l'homme et des principes démocratiques. Nous et l'UE évoquons régulièrement ces questions importantes avec le gouvernement d'Israël. L'article 38 concerne les territoires auxquels s'applique l'accord : comme il s'applique au territoire de l'État d'Israël, il ne doit pas inclure les Territoires occupés. La question de savoir si Israël commettrait une infraction à la Convention, en certifiant les marchandises produites dans les Territoires occupés comme étant des produits israéliens, est une affaire du ressort de la Commission (cls. 474-5). 
45.Lettre de Jeremy Hanley, membre du Parlement et ministre d'État aux Affaires étrangères et au Commonwealth Office de l'époque, datée de mars 1997 et adressée à Mark Lennox, membre du Parlement. Lecture de cette lettre a été faite devant la Chambre durant un débat ultérieur : Hansard, 17 décembre 1997 c. 462.

46.Des extraits de la discussion qui suivit ont été publiés dans un article (voir Shammas, 1999).

47.ES-10/3 du 15/7/97 ; ES 10/4 du 13/11/97 ; ES 10/5 du 17/3/98 ; ES 10/6 du 9/2/99. 48.En juin 1998, des organisations palestiniennes et internationales des droits de l'homme ont adressé au gouvernement suisse une « Lettre ouverte » exprimant leurs inquiétudes à ce sujet; le Centre palestinien pour les droits de l'homme, situé dans la ville de Gaza, a publié une série de communiqués de presse et de notes sur la question. 49.Al Gore, vice-président des États-Unis, aurait dit, s'adressant à une réunion de l'AIPAC : « Nous œuvrerons ardemment pour que la réunion proposée pour le 15 juillet n'ait pas lieu... L'Amérique la boycottera et incitera les autres à en faire de même. » Phyllis Bennis dans Middle-East International du 30/7/99.

50.Amnesty International a exhorté tous les États à participer à la conférence « et à examiner les mesures à prendre pour pousser Israël à respecter les obligations qu'il a contractées en vertu de la $\mathrm{IV}^{\mathrm{e}}$ Convention de Genève (déclaration publique 15/37/99 du 11 mai 1999) ; Human Rights Watch a écrit au président Clinton, appelant les États-Unis à appuyer la tenue de la conférence et déclarant que : « Il est déconcertant qu'un État signataire de la Convention ne veuille pas participer, mais il est déraisonnable que quelqu'un essaie d'entraver cette tentative de mise en œuvre de la Convention. » (Communiqué de presse du 12 juillet 1999) ; les organisations palestiniennes des droits de l'homme ont tenu une conférence parallèle à Genève les 14 et 15 juillet.

51.UN Doc. E/CN.4/2001/121 para. 114.

52.Ibid, para. 115 .

53.Palestine and the UN, Volume 6, issue 4.

54. « Nous pensons qu'en pareilles circonstances, la surveillance acceptée par les deux parties servirait leurs intérêts en mettant en œuvre le Rapport Mitchell. » Déclaration sur le Moyen-Orient, conclusions de la réunion des ministres des Affaires étrangères du Groupe des 8, 19 juillet 2001 à Rome (Texte intégral sur le site : http :www.g7.utoronto.ca/g7/foreign/fm091901_mideast.html). 55.UN Doc.A/56/440, 4 octobre 2001, Rapport du Rapporteur spécial de la Commission des droits de l'homme sur la situation des droits de l'homme dans les Territoires palestiniens occupés par Israël depuis 1967. 56.Résolution 1405 d'avril 2002.

INDEX

Mots-clés : Palestine, processus de paix, loi 
AUTEUR

LYNN WELCHMAN

Centre of Islamic and Middle East Law

School of Oriental and African Studies, Université de Londres 